\title{
Sequential Injection Titration with Spectrophotometric Detection for the Assay of Acidity in Fruit Juices
}

\author{
Jaroon JAKMUnEe, ${ }^{*}{ }^{*} * \dagger$ Thitima RUJIRALAI, ${ }^{*}$ and Kate GrUdPAN*,** \\ *Department of Chemistry, Faculty of Science, Chiang Mai University, Chiang Mai 50200, Thailand \\ **Institute for Science and Technology Research and Development, Chiang Mai University, \\ Chiang Mai 50200, Thailand
}

\begin{abstract}
A simple sequential injection analysis (SIA) with spectrophotometric detection for an assay of acidity in fruit juice was investigated. An alkaline reagent (sodium hydroxide), a sample and an indicator (phenolphthalein) were first aspirated and stacked as adjacent zones in a holding coil. With flow reversal through a reaction coil to the detector, zone penetration occurred, leading to a neutralization reaction that caused a decrease in the color intensity of the indicator being monitored for absorbance at $552 \mathrm{~nm}$. The effects of various parameters were studied. Linear calibration graphs for acidities of $0.2-1.0$ and $0.5-2.5 \% \mathrm{w} / \mathrm{v}$ citric acid as a standard, with a relative standard deviation of $1 \%$ (acidity of 0.3 -

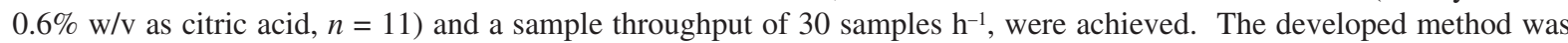
validated by a standard titrimetric method for assaying the acidity of fruit juice samples.
\end{abstract}

(Received September 8, 2005; Accepted November 12, 2005)

\section{Introduction}

Acidity serves many functions in food processing, such as preservative, flavoring agent, buffer and synergist. The acidity of a fruit juice is associated with the contents of several organic acids, which may naturally be present in fruit, or may be added during the manufacturing process. Citric acid is usually a major component, and thus the total acidity is usually expressed as the citric acid content.

Several methods have been applied to the determination of acidity, e.g., classical volumetric titration, ${ }^{1}$ spectrophotometry, ${ }^{2}$ ion chromatography, ${ }^{3}$ gas chromatography, ${ }^{4}$ high-performance liquid chromatography ${ }^{5}$ and capillary electrophoresis. ${ }^{6,7}$ These methods may involve tedious procedures, time/reagents consuming or expensive instruments, although some of them may provide information on the contents of different acids. Flow injection analysis (FIA) is usually applied for the rapid determination of total acidity employing different chemistries and detection systems. ${ }^{8-10}$ A simple conductometer or spectrophotometer has also been utilized by using ammonia or alkaline phenolphthalein as a reagent. ${ }^{10}$ However, a continuous flow feature of FIA consumes extensive amounts of reagents. Recently, sequential injection analysis (SIA) has been developed to avoid this problem and to provide additional advantages. ${ }^{11}$ SIA methods for the determination of citric acid were developed, but it concerns a complicated instrument. ${ }^{12-14}$

In this work, a SIA system employing an alkaline phenolphthalein reagent with simple cost-effective spectrophotometric detection was investigated for the assay of acidity in fruit juice samples.

$\dagger$ To whom correspondence should be addressed.

E-mail: scijjkmn@chiangmai.ac.th

\section{Experimental}

Chemicals

All chemicals were of analytical reagent grade, and deionized water was used throughout. Citric acid monohydrated (Carlo Erba) was used to preparation of standard solutions of different acidity values. Sodium hydroxide (EKA Nobel) and phenolphthalein (Merck) were used to prepare reagent solutions.

\section{SIA manifold}

A laboratory-made SIA analyzer (Center for Biotechnology, University of Turku, Finland) consisting of a syringe pump, a holding coil $(200 \mathrm{~cm}), 2$ of six port selection valves, a reaction coil $(100 \mathrm{~cm})$, a simple spectrophotometer (Spectronic 21, Bausch \& Lomb, USA) with a $10 \mathrm{~mm}$ path length flow-through cell (Hellma, Germany) and a personal computer was used. Figure 1 shows a diagram of the SIA manifold used. All tubings were $0.6 \mathrm{~mm}$ i.d. Teflon. An in-house software (AnalySIA, Center for Biotechnology, University of Turku, Finland) was used to control the system and to collect data from the detector via a plug-in interface card (Lab PC+, National Instruments, USA). The analysis was performed automatically following sequences that were written as a computer program called an AnalySIA script language (ASL).

Firstly, all of the tubings were cleaned by washing with deionized water. Then, sample or standard solutions, and the reagents were drawn to fill the sample or standard and reagent lines, respectively. The sequences for determining acidity were carried out as follows. First, the syringe of the pump was filled with $1500 \mu \mathrm{l}$ of the carrier solution (water). Then, $30 \mu \mathrm{l}$ of phenolphthalien and $50 \mu \mathrm{l}$ of sodium hydroxide solutions were sequentially aspirated from the valve SV2 into a holding coil. Next, $50 \mu \mathrm{l}$ of a sample or standard solution was inserted via the 


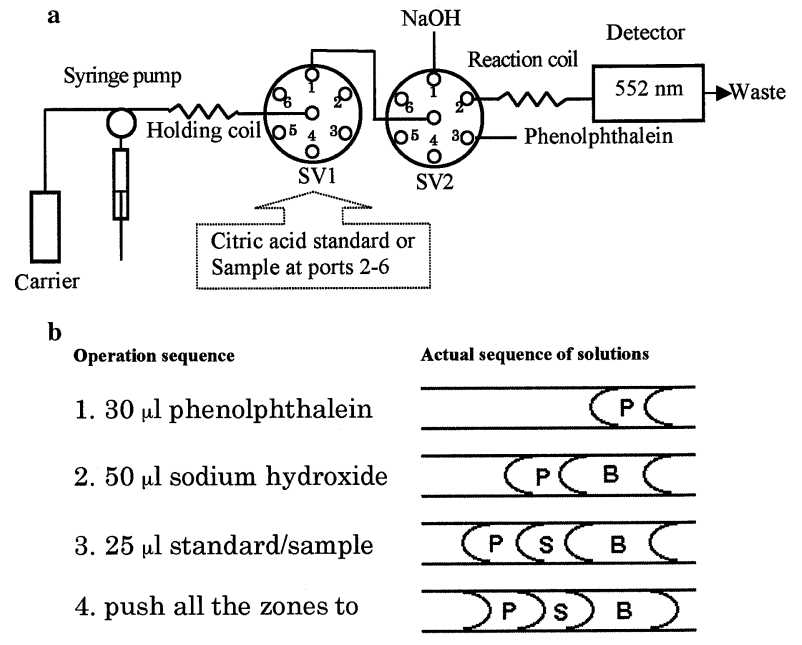

Fig. 1 (a) SIA manifold; SV1, and SV2 are selection valves 1 and 2, respectively. (b) SIA operation sequences; $\mathrm{P}$, phenolphthalein, $\mathrm{S}$, standard/sample; and B, sodium hydroxide solution.

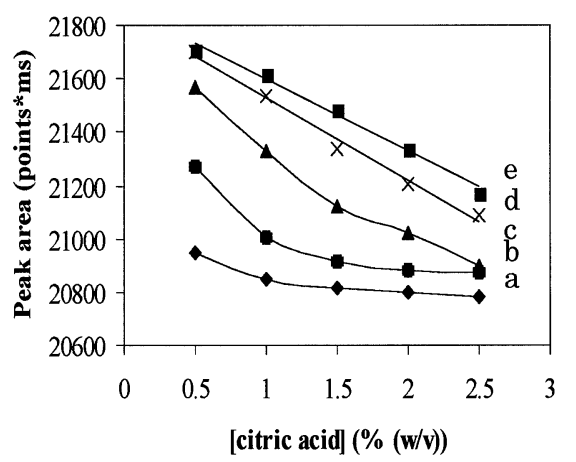

Fig. 2 Effect of the sodium hydroxide concentration. (a) 0.025, (b) 0.050 , (c) 0.100 , (d) 0.150 and (e) $0.200 \mathrm{M}$ (For the conditions, see the text; $r^{2}$ for lines $d$ and e are 0.9906 and 0.9909 , respectively).

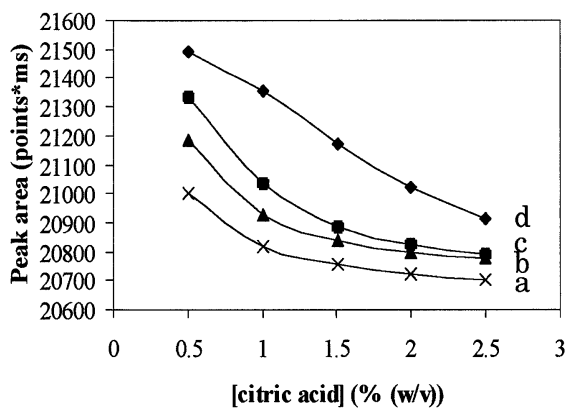

Fig. 3 Effect of the standard/sample volume. (a) 100, (b) 75, (c) 50 and (d) $25 \mu \mathrm{l}$ (For the conditions, see the text; $r^{2}$ for line $\mathrm{d}$ is 0.9950 ).

SV1 in between the two previous zones at about the interface of the zones. The sequence of zone became phenolphthalein: sample: sodium hydroxide. Finally, the zones were propelled through a mixing coil to a detector, while the transmission at $552 \mathrm{~nm}$ was monitored. The peak area was evaluated from the obtained SIA profile. The calibration graph was a plot of the peak area versus the acidity contents, expressed as the citric acid concentrations.
Table 1 Optimization ranges and suitable conditions for the SIA parameters used for determining of acidity in fruit juices

\begin{tabular}{lcc}
\hline \multicolumn{1}{c}{ Parameter } & $\begin{array}{c}\text { Optimization } \\
\text { range }\end{array}$ & $\begin{array}{c}\text { Suitable } \\
\text { condition }\end{array}$ \\
\hline Sodium hydroxide concentration $(\mathrm{M})$ & $0.025-0.200$ & 0.150 \\
Volume of sodium hydroxide solution $(\mu \mathrm{l})$ & $25-100$ & 50 \\
Phenolphthalein concentration $(\%(\mathrm{w} / \mathrm{v}))$ & $0.025-0.150$ & 0.050 \\
Volume of phenolphthalein solution $(\mu \mathrm{l})$ & $25-50$ & 30 \\
Sample volume $(\mu \mathrm{l})$ & $25-100$ & 25 \\
Flow rate $\left(\mu \mathrm{l} \mathrm{s}^{-1}\right)$ & $130-200$ & 150 \\
\hline
\end{tabular}

\section{Results and Discussion}

\section{Optimization}

The effects of the aspiration sequences of the standard/sample and the reagents, the concentrations and volumes of the reagents, volume of the standard/sample, and the flow rate of transporting solutions to the detector were investigated. Conditions that provided good slopes and linearity calibration graphs for the acidity contents in the ranges of $0.2-1.0$ and 0.5 $-2.5 \%$, which are normally found in samples, were considered to be appropriate. The obtained optimization ranges and suitable conditions are summarized in Table 1.

The effects of the concentrations of $\mathrm{NaOH}$ in the range of $0.025-0.200 \mathrm{M}$ were studied. A $50-\mu \mathrm{l}$ volume of $0.1 \%(\mathrm{w} / \mathrm{v})$ phenolphthalein, $50 \mu \mathrm{l}$ of $\mathrm{NaOH}$ and $25 \mu \mathrm{l}$ of citric acid standard solutions were sequentially aspirated before sending the zones to a detector. As can be seen from Fig. 2, 0.150 M $\mathrm{NaOH}$ provided a linear calibration graph. The effect of the volume of a $\mathrm{NaOH}$ solution $(25-100 \mu \mathrm{l})$ was investigated. It indicated that $50 \mu \mathrm{l} \mathrm{NaOH}$ should be suitable.

Phenolphthalein concentrations $(0.025-0.150 \%(\mathrm{w} / \mathrm{v}))$ were tried by aspirating $50 \mu \mathrm{l}$ of phenolphthalein, $50 \mu \mathrm{l}$ of $0.150 \mathrm{M}$ $\mathrm{NaOH}$ and $25 \mu \mathrm{l}$ of citric acid standard solutions. The lowest phenolphthalein concentration that significantly promoted the signal was $0.05 \%(\mathrm{w} / \mathrm{v})$. Among the volumes of phenolphthalein solutions investigated $(25-50 \mu \mathrm{l}), 30 \mu \mathrm{l}$ provided the best results.

Figure 3 shows the influence of standard/sample volumes (25 - $100 \mu \mathrm{l}$ ). It was found that $25 \mu \mathrm{l}$ of the standard/sample gave a linear calibration graph for $0.5-2.5 \%$ acidity. A further study pointed out that a linear calibration graph with a higher slope for $0.2-1.0 \%$ acidity could be achieved by using $50 \mu \mathrm{l}$ of the standard/sample solution.

The flow rate $\left(130-200 \mu \mathrm{l} \mathrm{s}^{-1}\right)$ for propelling the stacked zones to a detector was investigated. A calibration graph having a higher slope, but less linearity was obtained when a lower flow rate was used. A flow rate of $150 \mu \mathrm{l} \mathrm{s}^{-1}$ was chosen.

Other aspiration sequences were also studied, as summarized in Table 2. The aspiration sequence of phenolphthalein: sodium hydroxide: citric acid standard/sample gave a calibration graph having a better slope and linearity.

\section{Verification of the developed SIA system}

Solutions of bromothymol blue (BTB) dye $(0.01 \% \mathrm{w} / \mathrm{v})$ and borate buffer of $\mathrm{pH} 10(0.1 \mathrm{M})$ were used to verify the developed SIA system, as follows.

\section{Sequence of solutions}

In order to evaluate the actual sequences of aspirated solutions, a volume of tubing connected between the 2 selection valves was evaluated by the spectrophotometric method. ${ }^{15} \mathrm{~A}$ 
Table 2 Study on aspiration sequences

\begin{tabular}{|c|c|c|c|c|c|c|c|c|}
\hline \multicolumn{2}{|c|}{ Sequence $^{\mathrm{a}}$} & \multicolumn{5}{|c|}{$\begin{array}{l}\text { Peak area (points) })^{b} \text { for } \%(w / v) \text { of } \\
\text { standard citric acid solution }\end{array}$} & \multirow{2}{*}{$\begin{array}{l}\text { Calibration graph } \\
\text { equation }\end{array}$} & \multirow{2}{*}{$r^{2}$} \\
\hline Aspiration & Zone & 0.5 & 1.0 & 1.5 & 2.0 & 2.5 & & \\
\hline $\mathrm{P} / \mathrm{B} / \mathrm{S}$ & $\mathrm{P} / \mathrm{S} / \mathrm{B}$ & 21500 & 21400 & 21200 & 21100 & 20900 & $y=-296 x+21700$ & 0.9949 \\
\hline $\mathrm{S} / \mathrm{P} / \mathrm{B}$ & $\mathrm{S} / \mathrm{B} / \mathrm{P}$ & 21700 & 21500 & 21400 & 21300 & 21300 & $y=-194 x+21700$ & 0.9889 \\
\hline $\mathrm{B} / \mathrm{S} / \mathrm{P}$ & $\mathrm{B} / \mathrm{P} / \mathrm{S}$ & 21700 & 21500 & 21300 & 21200 & 21100 & $y=-282 x+21800$ & 0.9723 \\
\hline
\end{tabular}

a. P, B and S represents phenolphthalein, sodium hydroxide, and citric acid standard, respectively. b. Mean of triplicate results.

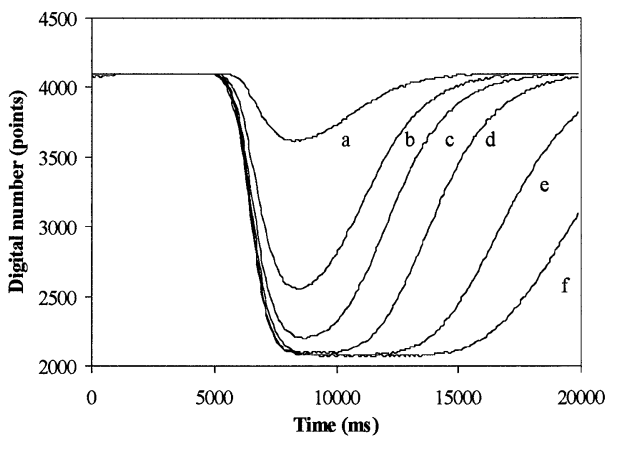

Fig. 4 SIA peak profiles of different volumes of dye solution aspirated into the SIA system. (a) 10, (b) 50, (c) 100, (d) 200, (e) 400 and (f) $600 \mu \mathrm{l}$ (For the conditions, see the text; digital number, a number obtained from an analog-to-digital converter unit, is directly proportional to the analog output signal from the detector).

series of known volumes $(0-200 \mu \mathrm{l})$ of BTB solution was pipetted into a $25 \mathrm{ml}$ volumetric flask and then diluted to the mark with a borate buffer solution. The same dye solution was aspirated to fill the tubing between the valves, and then pushed into a $25 \mathrm{ml}$ volumetric flask. After diluting with a borate buffer solution, the solution was taken to measure for the absorbance, and a calibration graph (plot of absorbance vs. volume of the dye solution) was constructed. The volume of tubing between the valves was examined from a calibration graph and found to be $50 \mu \mathrm{l}$, which would agree with the actual sequences of solutions ( $30 \mu \mathrm{l}$ of phenolphthalein, $25 \mu \mathrm{l}$ of sample and $50 \mu \mathrm{l}$ of sodium hydroxide) for the operational sequences described earlier (Fig. 1(b)).

\section{Precision of aspiration volume}

To study the precision of the aspiration volume, a programmed preset volume $(30-100 \mu \mathrm{l})$ of BTB was aspirated and transferred into a $25 \mathrm{ml}$ volumetric flask. After adjusting the volume to the mark with a borate buffer solution, the solution was taken to measure the absorbance and examined for the actual volume, as mentioned above. The relative standard deviations of triplicate results of all the volumes studied were less than $0.1 \%$.

\section{Effect of volume on dispersion}

A $200 \mu \mathrm{l}$ borate buffer, various volumes of BTB $(10-600 \mu \mathrm{l})$ and $200 \mu \mathrm{l}$ borate buffer were sequentially aspirated, and then propelled to a detector. The obtained recorded profiles, depicted in Fig. 4, exhibit the same height for a volume of 200 $\mu l$ or more, while wider peaks are observed. This indicates that a zone volume of more than $200 \mu \mathrm{l}$ may completely separate solutions on the two sides of the zone bolus, thus preventing any mixing of them together.

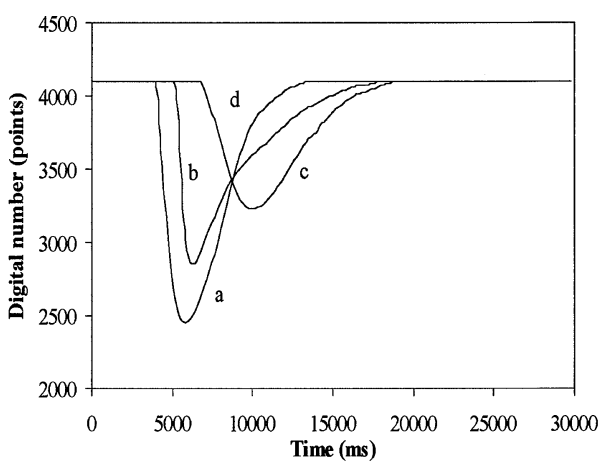

Fig. 5 Overlay peak profiles of the BTB dye solution as: (a) sodium hydroxide, (b) standard/sample, (c) phenolphthalein zones and (d) an overlapping zone (For the conditions, see the text; digital number, a number obtained from an analog-to-digital converter unit, is directly proportional to the analog output signal from the detector).

\section{Investigation of zone penetration}

Zone penetration was studied by using the conditions given in Table 1, except that the reagents and sample solutions were replaced by a borate buffer solution or a BTB solution. The borate buffer solution was also used as a carrier. The investigated zone was replaced with a BTB while the others were replaced with a borate buffer solution. The overlay recorded profiles, using BTB for replace each reagent and sample, are represented in Fig. 5. The overlap area (d in Fig. 5) of the profiles should indicate zone penetration, which would yield a reaction product.

\section{Precision of the procedure}

The precisions of the procedure were investigated for the analysis of standard solutions $(0.2-1.0 \% \mathrm{w} / \mathrm{v}$ citric acid $)$ and fruit juice samples. The relative standard deviations $(n=11$ for each concentration) were up to $1.0 \%$ for standard solutions and samples of fruit juices (containing acidities (as citric acid)) of $0.3-0.6 \%$ (Table 3), respectively. A sample throughput of 30 $\mathrm{h}^{-1}$ could be achieved.

\section{Analysis of fruit juice samples}

The developed procedure was applied to canned fruit juice samples, which were commercially available locally. The samples were filtered and/or diluted before subjecting to an assay by the developed SIA procedure and a conventional volumetric titration, ${ }^{1}$ as presented in Table 3 . The results from the two methods were not significantly different (applying the $t$ test at the $99 \%$ confident level). ${ }^{16}$

\section{Acknowledgements}

The Thailand Toray Science Foundation, the Thailand Research 
Table 3 Assay for the acidities of fruit juice samples

\begin{tabular}{lccc}
\hline \multirow{2}{*}{\multicolumn{1}{c}{ Sample }} & \multicolumn{2}{c}{ Citric acid found $(\%(w / v))$} & \\
\cline { 2 - 3 } & Titration $^{\mathrm{a}}$ & Proposed SIA $^{\mathrm{b}}$ & \\
\hline A (Lychee) & $0.34 \pm 0.00$ & $0.32 \pm 0.01$ & -5.9 \\
B (Apple) & $0.38 \pm 0.00$ & $0.37 \pm 0.00$ & -2.6 \\
C (White grape) & $0.46 \pm 0.00$ & $0.44 \pm 0.01$ & -4.4 \\
D (Valencia orange) & $0.58 \pm 0.01$ & $0.55 \pm 0.01$ & -5.2 \\
E (Tangerine orange) & $0.66 \pm 0.01$ & $0.64 \pm 0.01$ & -3.0 \\
F (Pineapple) & $0.42 \pm 0.00$ & $0.39 \pm 0.01$ & -7.1 \\
G (Lemon tea) & $0.44 \pm 0.01$ & $0.45 \pm 0.00$ & +2.3 \\
\hline
\end{tabular}

a. Mean of triplicate results.

b. Mean of 11 replicate results.

c. $\%$ Difference $=\left(\frac{\text { SI value }- \text { Titration value }}{\text { Titration value }}\right) \times 100$.

Fund (TRF) and the Postgraduate Education and Research Program in Chemistry (PERCH) are greatly acknowledged for their support. Dr. Rolf Sara, Dr. Michael Vilen, and Center for Biotechnology, Abo Akademi University and University of Turku, Finland are also very gratefully acknowledged for the SIA instrument on-loan for use in this work.

\section{References}

1. P. Cunniff, "AOAC Official Methods of Analysis", 1995, 16th ed., AOAC, Arlington.
2. H. E. Indyk and A. Kurmann, Analyst, 1987, 112, 1173.

3. G. Succani, S. Gherardi, A. Trifiro, C. S. Bordini, M. Calza, and C. Freddi, J. Chromatogr., A, 1995, 706, 395.

4. T. J. Barden, M. Y. Croft, E. J. Murby, and K. J. Wells, J. Chromatogr., A, 1997, 785, 251.

5. Q. C. Chen, S. F. Mou, K. N. Liu, Z. Y. Yang, and Z. M. Ni, J. Chromatogr., A, 1997, 771, 15.

6. W. C. Yang, A. M. Yu, Y. Q. Dai, and H. Y. Chen, Anal. Chim. Acta, 2000, 415, 75 .

7. L. Saavedra, A. Garcia, and C. Barbas, J. Chromatogr., A, 2000, 881, 395.

8. J. M. Alamo, A. Maquieira, R. Puchades, and S. Sagrado, Fresenius J. Anal. Chem., 1993, 347, 293.

9. K. Matsumoto and T. Tsukatani, Anal. Chim. Acta, 1996, $321,157$.

10. K. Grudpan, P. Sritharathikhun, and J. Jakmunee, Anal. Chim. Acta, 1998, 363, 199.

11. J. Ruzicka and G. D. Marshall, Anal. Chim. Acta, 1990, 237, 329 .

12. M. J. A. Canada and B. Lendl, Anal. Chim. Acta, 2000, $417,41$.

13. J. Jakmunee, L. Pathimapornlert, S. K. Hartwell, and K. Grudpan, Analyst, 2005, 130, 299.

14. J. F. van Staden, M. G. Mashamba, and R. I. Stefan, Talanta, 2002, 58, 1109.

15. J. Ruzicka and E. H. Hansen, "Flow Injection Analysis", 1988, 2nd ed., John Wiley \& Sons, New York.

16. G. D. Christian, "Analytical Chemistry", 2004, 6th ed., John Wiley \& Sons, Danvers, 97. 